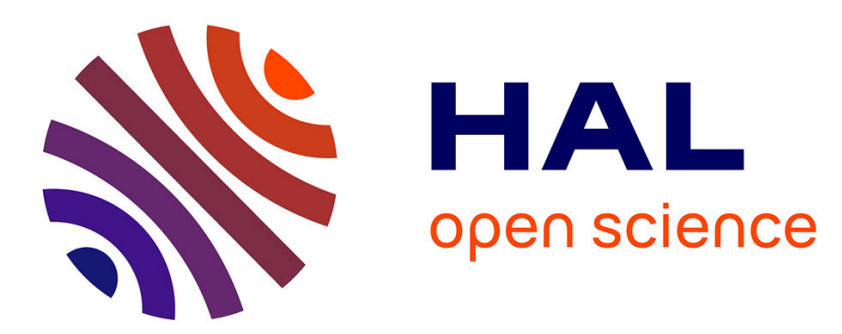

\title{
Les camionnettes de livraison dans les rues de bordeaux: de nouvelles études de déplacements urbains
}

\author{
Danièle Patier
}

\section{To cite this version:}

Danièle Patier. Les camionnettes de livraison dans les rues de bordeaux: de nouvelles études de déplacements urbains. Les Annales de la Recherche Urbaine, 1998, 78, pp.103-108. halshs-00325197

\section{HAL Id: halshs-00325197 \\ https://shs.hal.science/halshs-00325197}

Submitted on 26 Sep 2008

HAL is a multi-disciplinary open access archive for the deposit and dissemination of scientific research documents, whether they are published or not. The documents may come from teaching and research institutions in France or abroad, or from public or private research centers.
L'archive ouverte pluridisciplinaire HAL, est destinée au dépôt et à la diffusion de documents scientifiques de niveau recherche, publiés ou non, émanant des établissements d'enseignement et de recherche français ou étrangers, des laboratoires publics ou privés. 


\title{
LES CAMIONNETTES DE LIVRAISON DANS LES RUES DE BORDEAUX
}

\author{
DE NOUVELLES ÉTUDES DE DÉPLACEMENTS URBAINS
}

Danièle Patier

D vivaient un million d'habitants, des mesures avaient dû être prises pour réglementer l'approvisionnement et la circulation des véhicules: les chars n'avaient pas le droit de rouler la nuit, les grands commerces devaient s'implanter en périphérie... Depuis bientôt 2000 ans beaucoup de problèmes inhérents à l'approvisionnement des grandes agglomérations sont restés sans solution. Le maintien de l'habitat et du commerce dans le centre-ville est nécessaire à la vie de la cité, mais la cohabitation des déplacements de personnes et de marchandises a souvent été traitée en termes de relations conflictuelles. Le maintien d'activités commerciales dans les centres-villes ne peut se faire qu'en pensant à la façon d'organiser leur approvisionnement.

En 1990, en France, il n'existait, pour penser les problèmes de transport de marchandises en ville, ni statistique adaptée, ni synthèse des connaissances partielles des opérateurs et acteurs concernés, ni outil méthodologique.

Quelques études avaient pourtant été réalisées dans les années 70. Le contexte économique était marqué alors par une forte augmentation de la population accompagnée d'une croissance rapide de la mobilité des personnes et des marchandises grâce à l'utilisation de l'automobile. La problématique se réduisait à peu près à la question: «comment rendre le transport de marchandises, en zone urbaine, le moins pénalisant possible pour la circulation des voitures particulières »? Cette problématique a provoqué, dans de nombreuses grandes agglomérations, un développement de réglementations limitant le tonnage et les périodes de livraison des véhicules «fret» autorisés à circuler dans les zones denses.

Il s'en est suivi la délocalisation des sites logistiques en périphérie des villes, la multiplication des centres de groupage-dégroupage dans ces mêmes zones, et le départ de certaines entreprises à l'extérieur de la ville. La croissance de la population urbaine, des prix fonciers dans les centres, de la mobilité des personnes et des marchandises et celle encore plus rapide de la circulation automobile, accompagnée de la construction de rocades autoroutières, ont alimenté l'étalement urbain.

Parallèlement, on assiste à un fantastique développement du transport routier, avec une nouvelle organisation en hub and spokes en particulier dans la messagerie; une réorganisation industrielle, basée sur un grand nombre de sites géographiquement éloignés, la compétition économique entraînant la réduction des stocks et l'apparition

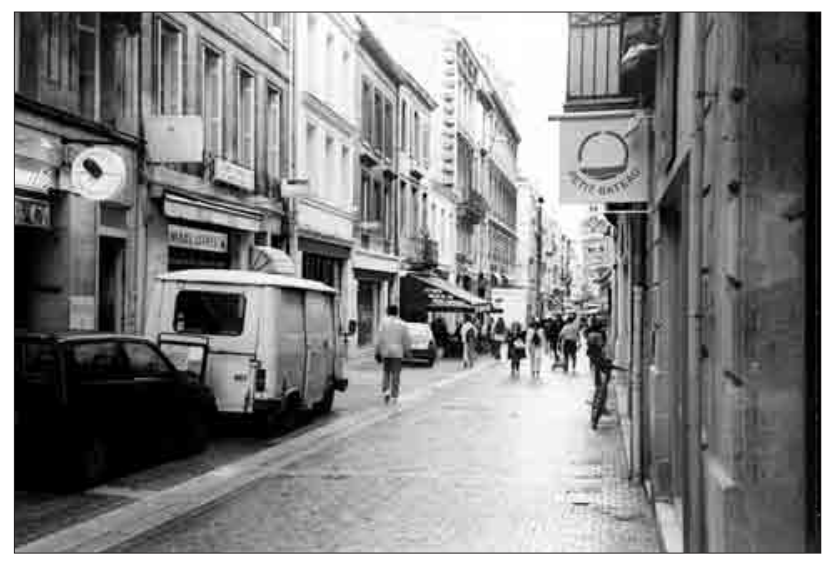

d'un fonctionnement en flux tendus, avec toutes les conséquences sur la multiplication des petits envois, la nécessité de rapidité, de flexibilité et de diversification des services logistiques.

Actuellement, la grande distribution hésite entre deux stratégies pour assurer son développement: augmenter l'attractivité des hyperconcentrations ou, au contraire, optimiser un réseau composé d'établissements hiérarchisés: Hypers, supers et supérettes. Or on sait qu'un super de centre-ville génère trois fois moins de voitures particulières qu'un hyper de périphérie pour la même quantité d'achat et que les flux «achats» représentent, en moyenne, $15 \%$ des flux de voitures particulières en ville. Une question importante et inattendue d'une politique de réduction de l'usage de la voiture en ville est donc de savoir quelles mesures la ville peut prendre pour que les choix logistiques de la grande distribution conduisent cette dernière à une stratégie «réseau» plutôt qu'à une

Les Annales de La Recherche Urbaine n ${ }^{\circ}$ 78, 0180-930-III-98/78/103/7 @ METL 
stratégie d'hyperconcentrations? Pour traiter un problème de transport de personnes, la ville aura à se pencher sur des questions de transport de marchandises et de foncier commercial. Elle aura, peut-être, à arbitrer systématiquement en faveur du transport de fret dans certains problèmes de partage de l'espace viaire, ce qui serait un retournement total de la politique des années 70 et suivantes.

Les années 90 marquent aussi la prise de conscience de l'importance du problème environnemental. En 1990, le Ministère des Transports et l'Agence de l'Environnement et de la Maîtrise de l'Energie font le constat des carences en la matière et lancent le programme national «TMV». La réflexion, sur la période 1970 - 1993 et la nature des préoccupations qui justifiaient le programme, nous ont amenés à construire celui-ci autour de l'interrogation essentielle suivante: «Quelle place ont les activités logistiques dans le fonctionnement du système urbain? En conséquence, comment la ville doit-elle les prendre en compte dans sa politique d'urbanisme et de déplacements urbains?».

C'est ainsi qu'en 1993 a été lancée une enquête d'envergure afin de constituer une base de données modèle sur une ville: l'enquête de Bordeaux, accompagnée de nombreuses études complémentaires sur les aspects qualitatifs du fonctionnement urbain et sur le jeu des acteurs, d'une étude critique du cadre législatif, réglementaire et institutionnel dans lesquels s'insèrent les activités de distribution urbaine des marchandises; enfin une étude sur la connaissance des coûts du transport de marchandises en ville mais aussi sur ce qui a pu se faire dans les pays voisins.

Le véritable déclic a été provoqué par les décisions récentes telle que la démarche visant à préparer les Plans de Déplacements Urbains à l'échelle des agglomérations, officialisée par la LOTI $^{1}$. La loi n ${ }^{\circ}$ 96-1236 du 30.12.1996 sur l'air et l'utilisation rationnelle de l'énergie dite «loi sur l'air» élargit le champ des PDU, et surtout rend obligatoire, dans un délai de deux ans, l'élaboration d'un PDU dans les agglomérations de plus de 100000 habitants. «Le plan de déplacements urbains définit les principes de l'organisation des transports de personnes et des marchandises, de la circulation et du stationnement, dans le périmètre de transports urbains.»

\section{Les flux urbains de marchandises}

En 1994, l'objectif premier de l'enquête quantitative de Bordeaux (750000 habitants), était de dresser un état des lieux, grâce à la constitution d'une base de données permettant de connaître les générateurs de trafics, l'importance des mouvements de véhicules transportant les marchandises, l'organisation de ces mouvements, les produits transportés...2

Elle a permis avant tout d'estimer le nombre de livraisons et enlèvements de marchandises générés par les acteurs économiques (établissements industriels, commerciaux et tertiaires) et de mettre en évidence des liens logiques entre les types d'activités et leurs localisations, le mode d'organisation du transport de marchandises lié à ces activités, les modes de gestion, les types de véhicules mis en jeu... et l'occupation de la voirie urbaine par les véhicules en stationnement et en circulation, à différentes périodes.

Deux enquêtes ont été réalisées en 1997, l'une à Dijon (220000 habitants), l'autre à Marseille (1020000 habitants) pour caler les résultats de Bordeaux et déceler l'existence de déterminants généraux de la logistique urbaine et de déterminants spécifiques à chaque ville ${ }^{3}$.

L'unité d'observation retenue comme la plus pertinente a été le mouvement, défini comme une réception, une expédition, ou une opération conjointe (réception et expédition), réalisé par un véhicule dans un établissement. Le mouvement permet d'appréhender la génération des principaux flux et ainsi de relier les activités économiques à l'encombrement qu'elles engendrent en agglomération. En effet, si les marchandises ont bien une origine et une destination identifiables, ce n'est pas le cas des véhicules qui les transportent. En milieu urbain, ces derniers effectuent plutôt des circuits complexes associant, lors d'une même tournée, un grand nombre de mouvements.

L'enquête tente d'appréhender la génération des trafics suivant trois axes principaux:

- estimation de l'occupation de la voirie par les véhicules de transport de marchandises, selon le type d'activités commerciales ou industrielles, ainsi que l'encombrement qu'ils génèrent;

- analyse des modes d'organisation (trace directe ou tournée), et de gestion (compte propre, compte d'autrui) du transport qui permettent l'acheminement des marchandises en ville;

- repérage des conditions de livraisons ou d'enlèvements des marchandises selon les modes de fonctionnement des entreprises.

Le périmètre d'enquête a été défini en collaboration avec les acteurs locaux. S'il n'a pas posé de problème majeur à Bordeaux et à Dijon, la proximité d'une commune importante près de Marseille (Aix-en-Provence), l'absence de syndicat de communes, et une situation géographique où s'imbriquent des flux à vocations différentes (présence du port, proximité des grandes zones industrielles et de stockage de Fos et Berre...) ont contribué à complexifier les choix. Chaque site fait l'objet d'une réflexion sur le périmètre qui prendra le mieux en compte l'approvisionnement et le fonctionnement des ses établissements industriels, commerciaux et tertiaire et le réseau viaire qui les dessert.

\footnotetext{
1. Article 28 de la loi n 82-1153 du 30 décembre 1982.

2. Cf. modélisation des transports de marchandises en ville: le modèle Freturb, J.-L. Routhier, P.-L. Aubert, Rome, 28-29 janvier 1998.

3. Hivert Laurent, INRETS, «Budgets Énergie, Environnement des déplacements dans I'agglomération bordelaise ».
} 
La méthodologie retenue est fondée sur le suivi des mouvements des véhicules induits par les livraisons ou enlèvements en zone urbaine. Ce suivi est réalisé à travers trois enquêtes:

1. - enquête auprès des «établissements » générateurs des déplacements de marchandises (industries, commerces, tertiaires). Un carnet de bord hebdomadaire permet le recensement de tous les mouvements de marchandises liés à l'activité de l'établissement pendant une semaine;

2. - enquête auprès des «chauffeurs-livreurs » ayant assuré les livraisons. Elle a permis, pour la première fois, de connaître le cheminement des produits, les différents arrêts lors des tournées, leur durée, les conditions de stationnement, les distances parcourues, les caractéristiques des véhicules... A Bordeaux, cette enquête était auto-administrée et retournée par la poste. Le nombre de retours de questionnaires (950), estimé un peu faible, a laissé planer quelques doutes sur certaines réponses peu fiables. C'est pourquoi la méthode d'investigation sur Marseille et Dijon a été nettement modifiée et renforcée. D'une enquête auto-administrée à Bordeaux, on est passé, à Dijon et Marseille, à une enquête en «face à face», et même dans le cas de tournées complexes à une enquête avec «enquêteur embarqué». Ces nouvelles procédures ont permis de renforcer considérablement la connaissance sur les parcours effectués.

3. - à Bordeaux, une enquête «transporteur» auprès des entreprises de transport public impliquées dans les tournées décrites par les chauffeurs a permis de comprendre l'organisation logistique des professionnels du transport.

Cette enquête a apporté, par rapport aux études antérieures:

- une amélioration de l'identification de l'activité et la nature du local de chaque établissement desservi pendant la tournée. Ce qui permet de lever l'hypothèse sur l'homogénéité des tournées;

- une meilleure connaissance des lieux de départ et fin de tournées;

- la description de tous les arrêts de la tournée avec précision sur le temps d'arrêt, les conditions de chargement, de stationnement, du taux de remplissage du véhicule à chaque arrêt;

- la décomposition de la distance parcourue par tronçon et temps d'arrêt à chaque positionnement;

- une meilleure prise en charge des km à vide, permettant de distinguer les traces directes et les tournées en fonction du nombre de trajets par rapport au nombre de livraisons et enlèvements;

- une connaissance complète de l'itinéraire permettant de lever l'ambiguïté sur les départs ou retours à vide et les éventuels passages par des plates-formes, (qui ont pu être oubliés dans la première enquête) et le type de plates-formes utilisées.
Le tracé des itinéraires sur un plan de la ville et de l'agglomération a été réalisé sur les trois sites. Il donne une vision parfaite des choix d'itinéraires et des nœuds critiques de circulation.

\section{La part du transport de marchandises dans le trafic motorisé urbain}

Les 1500 établissements du secteur privé enquêtés à Bordeaux ont occasionné 270000 livraisons et enlèvements en une semaine auxquels il faut ajouter de l'ordre de 100000 mouvements réalisés dans le cadre du fonctionnement des services publics (services postaux, chantiers, déménagements, collecte des déchets...) qui ont pu être dénombrés par des enquêtes complémentaires ou des estimations.

Il est important d'intégrer aux transports de marchandises les déplacements des véhicules particuliers effectués pour le motif achat. En effet, toute modification en termes de localisation ou d'organisation des commerces peut entraîner une substitution entre les modes de gestion et d'organisation des livraisons ou enlèvements. Par exemple, un développement des moyennes surfaces en centre-ville ou encore le développement des livraisons à

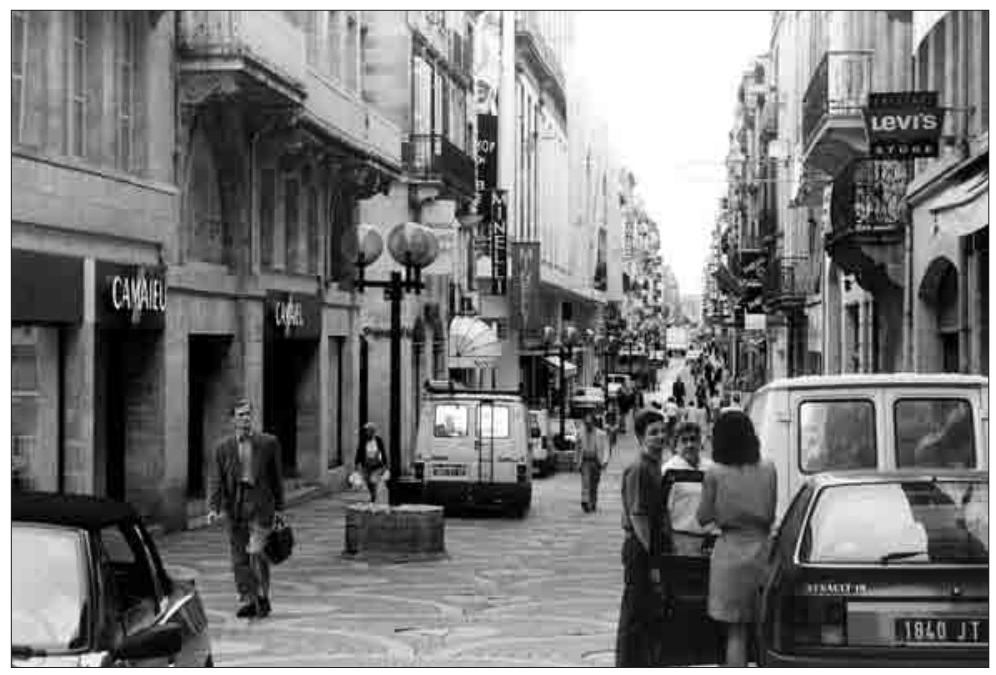

omicile ont un impact direct sur les déplacements pour motif d'achat.

Les opérations de livraisons et enlèvements de marchandises recensées lors de l'enquête de Bordeaux représentent $41 \%$ des flux (estimés en «véhicules-kilomètres-en équivalents-voiture particulière») et les déplacements d'achats des citadins, $40 \%$. Ces proportions peuvent varier d'une ville à l'autre selon leurs spécificités économiques.

D'après les études du Laboratoire d'Économie des Transports et du Centre d'Études techniques de l'Équipement, l'encombrement dû aux véhicules de chantier, 
mesuré dans cette même unité (équivalent VP) est de $5 \%$, celui dû aux déménagements de $5 \%$, celui dû à l'enlèvement des déchets de $3 \%$. Transports de marchandises et transports de personnes sont à peu près équivalents et forment l'essentiel de la congestion urbaine.

A Bordeaux, on compte 2300000 déplacements de personnes par jour dont $62 \%$ sont effectués en voiture particulière, et parmi ces derniers $10 \%$ pour faire des

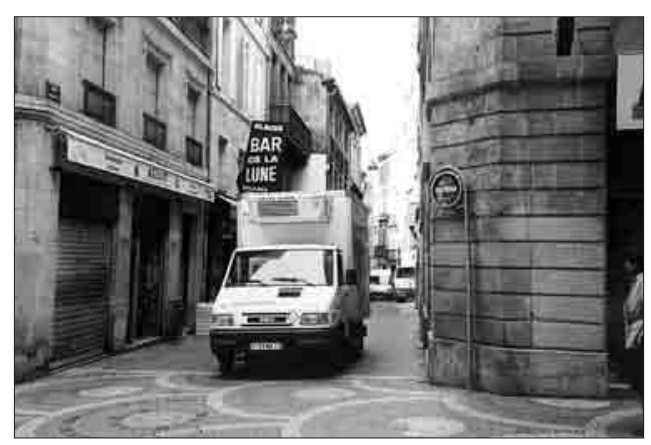

courses. Les livraisons sont au nombre de 300000 par jour environ, mais donnent toutes lieu à des déplacements motorisés, plus longs que les voyages de personnes, et dans des véhicules plus grands; l'encombrement est plus important. L'étude évoquée ici présente évidemment des calculs plus précis que ceux qu'on peut retenir dans le cadre limité d'un article.

\section{Deux fois plus d'opérations de livraisons que d'enlèvements}

Cette prédominance des livraisons par rapport aux enlèvements se reproduit dans les trois villes dans la proportion de $66 \%$ de livraisons pour $34 \%$ d'enlèvements.

$73 \%$ des parcours sont effectués en trace directe pour un seul paquet et ne réalisent que $24 \%$ des livraisons/enlèvements. A l'inverse, $27 \%$ des parcours, cette fois relevant de tournées, assurent $76 \%$ des livraisons/enlèvements.

Si le professionnel rentabilise d'un point de vue logistique le déplacement qu'il effectue en tournée, expéditeurs et destinataires dans le cas de la trace directe ne font que répondre à une logique commerciale (pas de stock, flux tendus); d'où la nécessité de multiples déplacements réalisés à la demande avec des véhicules légers qui ne font qu'une livraison par trajet. Par ailleurs, le véhicule se gare en double file ou sur un trottoir pour gagner du temps...

Il faut toutefois faire la distinction entre l'entreprise industrielle ou de distribution qui, ayant son propre service transport, organise ses livraisons de façon très professionnelle, et l'artisan ou le petit commerçant qui agit au coup par coup. Les destinataires sont essentiellement organisés en trace directe, les expéditeurs, et évidemment, les transporteurs, plutôt en tournées.

\section{Une gestion basée sur le transport en compte propre}

A Bordeaux et Marseille les deux-tiers des livraisons et enlèvements sont effectués en compte propre (destinataires et expéditeurs des marchandises), alors que les professionnels du transport n'en assurent que le tiers. A Dijon le partage est équitable entre compte propre et compte d'autrui, ce qui marque la spécificité d'une ville plutôt tertiaire.

Industrie, grands magasins et activités tertiaires font majoritairement appel au compte d'autrui. C'est le commerce de détail qui est le plus concerné par le compte propre expéditeur. A l'inverse, les artisans présentent la plus forte proportion de compte propre destinataire. Ceux-ci vont en effet fréquemment s'approvisionner auprès d'un commerce de gros ou d'une grande surface, puis se rendent directement à leur atelier ou sur un chantier.

\section{RÉPARTITION DES MOUVEMENTS SELON L'ACTIVITÉ}

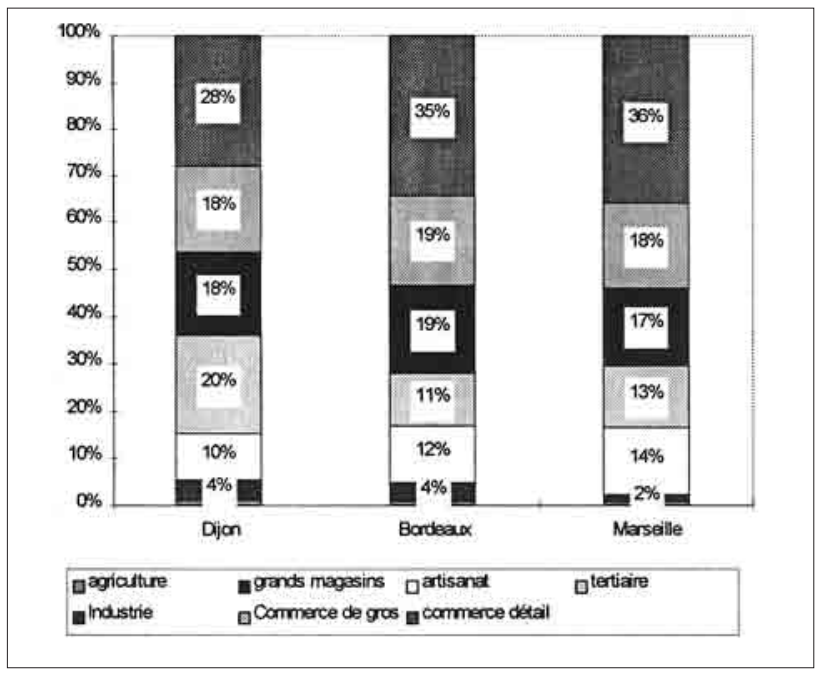

\section{Le commerce de détail, véritable moteur de la ville}

Le commerce de détail à lui seul occasionne le tiers des livraisons et enlèvements. Les expéditeurs dans cette activité réalisent $97 \%$ des livraisons. Si l'on tient compte de toutes les activités liées au commerce (commerce de gros, grands magasins, et artisanat) la proportion passe alors à $70 \%$ des opérations. A Bordeaux, $22 \%$ des livraisons sont réalisées au centre-ville où sont concentrés les commerces, sur $2 \%$ de la superficie totale de l'agglomération. Ainsi l'hyper-centre est très congestionné avec 20000 livraisons ou enlèvements au $\mathrm{km}^{2}$. Dans cette même zone on peut dénombrer plus de 10000 emplois au $\mathrm{km}^{2}$. La particularité du commerce de détail tient dans la prise en 
charge massive des livraisons et enlèvements par le mode de transport en compte propre et particulièrement par les expéditeurs eux-mêmes. Les expéditeurs réalisent $97 \%$ des livraisons lors de tournées à l'aide de véhicules légers (82\% d'entre eux font de moins de 3,5 tonnes, $70 \%$ sont des camionnettes, et $12 \%$ de voitures particulières). Les camionnettes stationnent principalement sur les trottoirs et en double file (pour la moitié des livraisons effectuées dans l'ensemble de l'agglomération mais pour $60 \%$ dans l'hyper-centre). Les infractions sont d'autant plus nombreuses que les véhicules sont plus grands. $76 \%$ des livraisons réalisées par les expéditeurs à l'aide de voitures particulières pratiquent ce type de stationnement. A l'inverse, les camions porteurs livrent dans $53 \%$ des cas de cette façon.

Les transporteurs, minoritaires dans ce secteur d'activité, utilisent des camions porteurs pour effectuer la moitié de leurs livraisons, $40 \%$ étant réalisées à l'aide de camionnettes. Dans l'hyper-centre ce sont encore $75 \%$ des livraisons effectuées par les camionnettes et $80 \%$ par les camions porteurs qui stationnent sur les trottoirs ou en double file.

\section{Les types de véhicules utilisés}

Le type de véhicule utilisé varie selon l'activité. Les camions porteurs et semi-remorques sont davantage utilisés dans les grands magasins et les entrepôts; les camionnettes dans l'activité du commerce de détail, le tertiaire, l'artisanat. Les deux-tiers des livraisons et enlèvements effectués par les entrepôts sont réalisés par des semi-remorques, tandis que le commerce de détail et le secteur tertiaire réalisent plus de la moitié de leurs livraisons/enlèvements à l'aide de camionnettes.

Pour une même activité, le type de véhicule utilisé dépend du mode de gestion. Le tiers des mouvements effectués par les destinataires se font en voiture particulière; plus de la moitié de ceux des expéditeurs avec des camionnettes et près de $40 \%$ des livraisons des entreprises de transports sont réalisées avec des camions porteurs.

Les véhicules ne dépassant pas 3,5 t de PTAC représentent près des deux-tiers des véhicules utilisés par les destinataires et les expéditeurs. Près des deux-tiers des livraisons et enlèvements sont effectués sans moyens de manutention. Dans la plupart des cas, les chauffeurs-livreurs estiment que les aménagements proches des établissements ne sont pas prévus pour leurs activités de chargement et déchargement.

\section{Mode de stationnement}

Dans l'ensemble de l'agglomération de Bordeaux, $35 \%$ des établissements disposent d'emplacements de livraison privés et seulement $6 \%$ disposent d'un emplacement réservé. Dans l'hyper-centre, $60 \%$ des établissements ne disposent
LE MODE DE STATIONNEMENT OFFERT AU LIEU DE LIVRAISON DANS L'AGGLOMÉRATION DE BORDEAUX

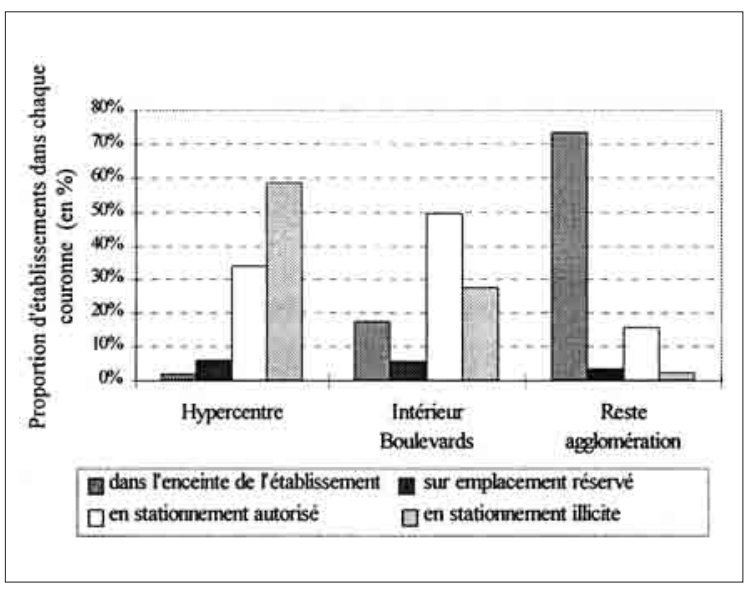

d'aucun emplacement de stationnement licite pour leurs livraisons. Le stationnement illicite est donc généralisé en centre-ville, du fait qu'à cet endroit, les emplacements privés sont très rares et les emplacements publics réservés aux livraisons sont fréquemment occupés par des véhicules en stationnement. Parmi les établissements qui ne disposent ni d'emplacement privé ni d'emplacement autorisé, $65 \%$ sont situés à proximité d'un emplacement autorisé payant ou non. Le tiers d'entre eux ne disposent d'aucun emplacement de stationnement autorisé.

Dans la pratique, la majorité des livraisons sont effectuées sur des lieux de stationnement illicite: sur l'agglomération tout entière, $68 \%$ des livraisons en l'absence d'emplacement spécifique sont effectuées en double file ou sur les trottoirs! La pratique du stationnement est fortement contrainte par la densité urbaine: en hyper-centre, alors que pour $32 \%$ des établissements, des emplacements sur voirie sont théoriquement disponibles, moins de $10 \%$ des livraisons les utilisent. Dans les zones moins denses, le stationnement illicite reste important: la pratique est acquise de se garer en double file ou sur les trottoirs, de manière à limiter la pénibilité et la durée du

RYTHMES HORAIRES DES LIVRAISONS/ENLÈVEMENTS ET DES DÉPLACEMENTS DE PERSONNES DANS L'AGGLOMÉRATION DE BORDEAUX

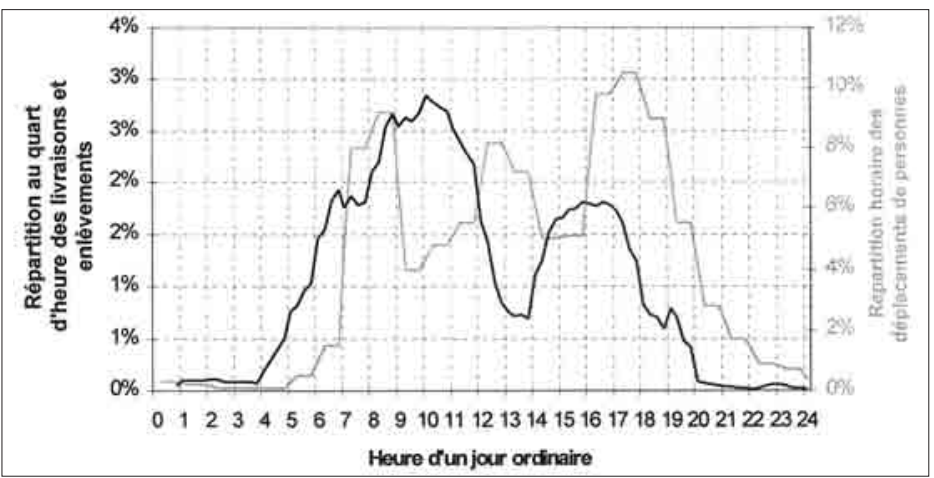

Sources : enquête Bordeaux, LET, étude BEED, INRETS 
déchargement, même s'il existe des places de stationnement à proximité.

Les heures de pointe du transport lié à la livraison ou l'enlèvement de marchandises en ville sont décalées par rapport à celles des déplacements de personnes. La plupart des livraisons se font pendant les heures d'ouverture des établissements. Une réflexion est en cours sur la possibilité de livrer hors la présence du destinataire. Cela va du sas déjà en service dans certaines professions jusqu'au dépôt dans des lieux spécifiques aménagés et gérés selon les filières de produits.

\section{L'usage des plates-formes}

L'enquête réalisée auprès des transporteurs à Bordeaux a permis de connaître la répartition des types de chaînes logistiques utilisées, dévoilant des configurations différentes lorsqu'il s'agit de transport de lot occasionnel par rapport à la messagerie et au lot régulier. Cette répartition reflète l'organisation logistique des professionnels du transport. Les entreprises de transport n'effectuent que le tiers de l'ensemble des livraisons/enlèvements en agglomération. Pour la messagerie et le lot régulier, l'approvisionnement de la ville s'effectue au moyen d'une vingtaine d'organisations logistiques différentes. On observe un fort usage des plates-formes (pour plus de la moitié des parcours), l'importance du centre-ville dans l'organisation logistique. Le type de chaîne le plus utilisé est celui qui relie l'agglomération à l'extérieur, en passant par une plate-forme.

Le transport de lot occasionnel repose sur une organisation plus simple basée sur neuf types de chaînes organisationnelles, dont la plus utilisée relie l'agglomération à l'extérieur, sans passage par une plate-forme. Plus des quatre-cinquièmes des tournées sont concentrées sur cinq types de chaînes dont la plupart concernent l'agglomération sans entrer au centre-ville.

\section{Perspectives}

Ces résultats alimentent le modèle de génération des flux de véhicules de marchandises en ville, modèle «FRETURB», mis au point au LET ${ }^{4}$. Ce modèle n'est pas conçu pour prédire des flux, mais pour simuler des effets de mesures prises en matière de réglementation, de planification et de logistique urbaines sur les livraisons et les encombrements de voirie qui en résultent. Ces recherches ont permis d'élaborer un guide méthodologique pour les agglomérations de plus de 100000 habitants afin d'intégrer les marchandises dans leur Plan de Déplacement Urbain dont la date limite d'élaboration est prévue pour la fin de l'année 98, avec une évaluation dans cinq ans ${ }^{5}$.

Déjà certaines agglomérations ont pu bénéficier de ces premiers acquis méthodologiques. Par exemple, à Lyon, une étude a été réalisée dans le quartier des Capucins (équivalent du Sentier à Paris). Elle a été réalisée avec la collaboration des différents acteurs, associations de commerçants, Chambre de Commerce, techniciens de la ville, urbanistes et responsables de la voirie et de la circulation. C'est aussi là que réside la nouveauté, dans cette concertation nécessaire entre tous les intéressés.

Aujourd'hui, l'ensemble des grandes agglomérations portent un intérêt croissant à ce thème. A des niveaux différents, chacune mène une réflexion sur la meilleure façon de gérer la logistique urbaine. Dans le cadre du programme PREDIT, une enquête sur les expériences concrètes dans le domaine du transport de marchandises a été menée auprès d'une trentaine d'agglomérations. Elle révèle la rareté des expériences innovantes, et l'insuffisance de la seule approche réglementaire. Une dizaine d'agglomérations sont prêtes à tenter une expériencepilote: mise en place de centres de distribution urbaine, utilisation de véhicules peu polluants, mise à disposition des achats effectués en centre-ville dans des consignes implantées dans les parkings localisés près de stations de tramway, promotion des outils d'échanges de données informatisées, aide à l'optimisation des tournées, etc.

Danièle Patier

\footnotetext{
4. Modélisation des transports de marchandises en ville: le modèle Freturb, Routhier Jean-Louis, Aubert Pierre-Louis, Rome, 28-29 janvier 1998.

5. "L'intégration des marchandises dans les P.D.U., guide méthodologique», CERTU, CRET, DRAST, ADEME, LET, L. Dablanc.
}

> Danièle Patier, spécialiste de l'économie des transports, développe actuellement ses recherches sur les transports urbains de marchandises dans le cadre du Laboratoire d'Économie des Transports à Lyon.

L'étude et sa bibliographie peuvent être consultés au Laboratoire d'Économie des Transports. La présentation donnée ici est très abrégée. 